\title{
Salivary Gland Hyalinizing Clear Cell Carcinoma
}

\author{
Jung-Chia Lin ${ }^{1} \cdot$ Jia-Bin Liao $^{1,2} \cdot$ Hsiao-Ting Fu ${ }^{1} \cdot$ Ting-Shou Chang ${ }^{2,3,4} \cdot$ Jyh-Seng Wang ${ }^{1,2,5}$ \\ 1Department of Pathology and Lab Medicine, Kaohsiung Veterans General Hospital, Kaohsiung; ${ }^{2}$ National Defense Medical Center, Taipei; \\ ${ }^{3}$ Department of Otolaryngology, Kaohsiung Veterans General Hospital, Kaohsiung; ${ }^{4}$ nstitute of Public Health, College of Medicine, National Cheng Kung University, Tainan; \\ ${ }^{5}$ Department of Medicine, National Yang-Ming University School of Medicine, Taipei, Taiwan.
}

Hyalinizing clear cell carcinoma (HCCC) is a rare malignant salivary gland tumor that was characterized as a distinct entity by Milchgrub et al. in 1994. ${ }^{1}$ It has a slight female predominance with ages ranging from 25 to 87 years (mean, 59.4 years). ${ }^{2}$ Most cases occur in the oral cavity, mainly the palate and tongue. ${ }^{2}$ Histologically, HCCC is characterized by the predominance of clear cells embedded in a characteristic dual hyaline and fibrocellular stroma. ${ }^{3}$ In 2011, Antonescu et al. ${ }^{2}$ identified EWSR1 rearrangements in this tumor, which have been subsequently observed in $82 \%$ of cases. This genetic change allows the distinction of HCCC from other salivary gland neoplasms with a clear cell phenotype. Here, we present a typical case of HCCC, which we believe is the first case report from Taiwan.

\section{CASE REPORT}

A 37-year-old female patient visited our clinic complaining of painless swelling on the ventral tongue that had been present for months. Intraoral examination revealed a $1 \times 1 \mathrm{~cm}$ nodule on the left ventral tongue and an excisional biopsy was done. Light microscopy showed an infiltrative tumor composed mostly of clear cells with spindle cell stroma. The tumor cells were immunoreactive for cytokeratin AE1/3 (CKAE1/3) and p63, and the spindle cell stroma for smooth muscle actin (SMA).

\section{Corresponding Author}

Jyh-Seng Wang, MD, PhD

Department of Pathology and Lab Medicine, Kaohsiung Veterans General Hospital,

386 Ta-Chung 1st Rd., Kaohsiung 813, Taiwan

Tel: +886-7-3422121 (ext 6318), Fax: +886-3422288,

E-mail: jswang@vghks.gov.tw

Received: March 27, 2015 Revised: April 20, 2015

Accepted: May 6, 2015
The original pathology report was myoepithelial carcinoma (MC) due to misinterpretation of the p63 immunoreactivity as myoepithelial differentiation. The diagnosis was soon revised to HCCC after recognizing this new entity.

The tumor comprised of cords and nests of tumor cells with skeletal muscle infiltration and perineural invasion (Fig. 1A). The tumor cells had clear and pale eosinophilic cytoplasm (Fig. 1B), fine nuclear chromatin with occasional small nucleoli, and no mitotic figure. The tumor cells were embedded in a characteristic dual hyaline and fibrocellular stroma (Fig. 1C). No ductal formation was seen. On immunohistochemical stains, the tumor cells were positive for CKAE1/3 and p63 (Fig. 1D), but negative for myoepithelial cell markers, such as SMA, CD10, S100, myosin, calponin, glial fibrillary acidic protein (GFAP), muscle specific actin (MSA), and desmin. The spindle cells in the fibrocellular stroma were positive for SMA and CD10 (Fig. 1E) but negative for CKAE1/3, p63, myosin, calponin, GFAP, MSA, S100, and desmin. The clear cells contained abundant glycogen highlighted by a diastase sensitive periodic acid-Schiff positive reaction (Fig. 1F, G). The result of the mucicarmine stain for mucin was negative. Fluorescence in situ hybridization for EWSR1 showed a break-apart signal pattern in 25\% of the tumor cells (Fig. 1H), confirming the presence of EWSR1 gene rearrangement. Since the margin was focally involved by the tumor, the patient received further excision with safe resection margin, which showed no residual tumor.

\section{DISCUSSION}

HCCC was first described by Milchgrub et al. in $1994^{1}$ as a rare salivary gland carcinoma made up of clear cells forming cords and nests in a hyalinized stroma. This tumor was often 

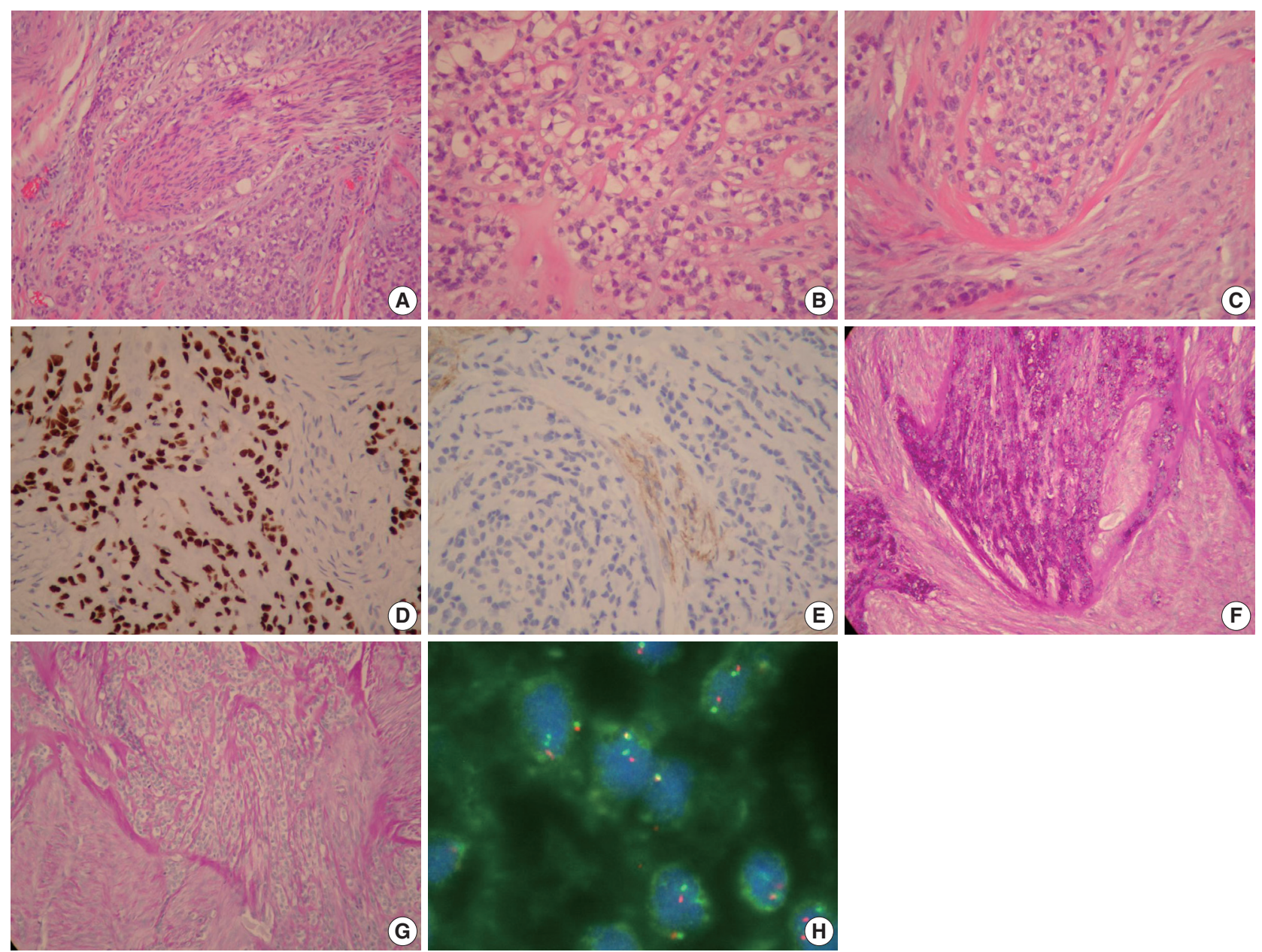

Fig. 1. (A) Hyalinizing clear cell carcinoma (HCCC) showing cords and nests of tumor cells with perineural invasion. The tumor cells have a clear cytoplasm (B) and are embedded in a characteristic dual hyaline and fibrocellular stroma (C). (D) Almost all clear cells are positive for p63. (E) The tumor cells are negative for CD10 while the myofibroblastic spindle cells in the stroma are positive for CD10. (F, G) Tumor cells with clear cytoplasm contain abundant glycogen which is positive for periodic acid-Schiff and sensitive to diastase digestion. $(\mathrm{H})$ Fluorescence in situ hybridization shows a break-apart signal pattern in 25\% of the tumor cells confirming the presence of EWSR 1 rearrangement.

confused with other clear cell mimics, such as epithelial-myoepithelial carcinoma (EMC), mucin-depleted mucoepidermoid carcinoma (MEC) and MC. It was separated from these entities because of its lack of apparent squamous, mucinous, and myoepithelial differentiation. ${ }^{1}$ HCCC is currently classified by the World Health Organization (WHO) as a diagnosis of exclusion: "clear cell carcinoma, not otherwise specified." ${ }^{, 4}$ Many small case series and case reports have been described, ${ }^{5-7}$ but none added new insights into HCCC until Antonescu et al. ${ }^{2}$ recently identified a consistent EWSR1-ATF1 fusion in HCCC.

In the differential diagnosis, EMC has a prominent ductal structure of tumor cells and does not show characteristic dual hyalinizing and fibrocellular stroma of HCCC. HCCC can be distinguished from MC by lack of myoepithelial cell markers such as S100, calponin, and SMA. Neither EMC nor MC has the EWSR1 gene rearrangement as seen in HCCC. Mucous cells may be absent in mucin-depleted MEC and is very difficult to differentiate from HCCC. However, mucin-depleted MEC lacks the characteristic dual stroma and EWSR1 rearrangement of HCCC. ${ }^{3}$ It should be noted that clear cell odontogenic carcinoma also has EWSR1 rearrangement as well as dual hyalinizing and fibrocellular stroma, and cannot be separated reliably from HCCC by the morphology or molecular marker, except by its location in the jaw bone. ${ }^{3,8}$

Treatment usually involves primary resection with negative margins. Cases of HCCC with recurrence or metastasis have been reported and proven by molecular analysis; however, the vast majority of HCCC have had good outcomes. ${ }^{3,4}$

In summary, we present a typical case of HCCC with characteristic dual stroma, lack of myoepithelial differentiation and 
presence of EWSR1 rearrangement. This is the first case report from Taiwan. Molecular genetic methods have become an integral part of modern oral pathology and play an important role in helping to categorize cases that defy traditional morphologic analysis. In the molecular era, "NOS" is no longer fitting for HCCC. The term "NOS" is at odds with the presence of the specific EWSR1-ATF1 fusion that clearly sets HCCC apart from other salivary neoplasia. Adding "hyalinizing" is also recommended, even though this feature is not present in all cases. The benefit of its inclusion is the mental association with a salivary clear cell malignancy. ${ }^{9}$

\section{Conflicts of Interest}

No potential conflict of interest relevant to this article was reported.

\section{REFERENCES}

1. Milchgrub S, Gnepp DR, Vuitch F, Delgado R, Albores-Saavedra J. Hyalinizing clear cell carcinoma of salivary gland. Am J Surg Pathol 1994; 18: 74-82.

2. Antonescu CR, Katabi N, Zhang L, et al. EWSR1-ATF1 fusion is a novel and consistent finding in hyalinizing clear-cell carcinoma of salivary gland. Genes Chromosomes Cancer 2011; 50: 559-70.

3. Weinreb I. Hyalinizing clear cell carcinoma of salivary gland: a review and update. Head Neck Pathol 2013; 7 Suppl 1: S20-9.

4. Ellis G. Clear cell carcinoma, not otherwise specified. In: Barnes L, Eveson JW, Reichart P, Sidransky D, eds. World Health Organization classification of tumours: pathology and genetics of head and neck tumors. Lyon: IARC Press, 2005; 227-8.

5. O'Sullivan-Mejia ED, Massey HD, Faquin WC, Powers CN. Hyalinizing clear cell carcinoma: report of eight cases and a review of literature. Head Neck Pathol 2009; 3: 179-85.

6. Solar AA, Schmidt BL, Jordan RC. Hyalinizing clear cell carcinoma: case series and comprehensive review of the literature. Cancer 2009; 115: 75-83.

7. Yang S, Zhang J, Chen X, Wang L, Xie F. Clear cell carcinoma, not otherwise specified, of salivary glands: a clinicopathologic study of 4 cases and review of the literature. Oral Surg Oral Med Oral Pathol Oral Radiol Endod 2008; 106: 712-20.

8. Bilodeau EA, Weinreb I, Antonescu CR, et al. Clear cell odontogenic carcinomas show EWSR1 rearrangements: a novel finding and a biologic link to salivary clear cell carcinomas. Am J Surg Pathol 2013; 37: 1001-5.

9. Brandwein-Gensler M, Wei S. Envisioning the next WHO head and neck classification. Head Neck Pathol 2014; 8: 1-15. 